\title{
ICA decreases myocardial hypertrophy injuries induced by isoproterenol by inhibiting endoplasmic reticulum stress
}

\section{Qiufang Zhang}

\author{
Department of Pharmaology, Department of Pharmaology, Hubei University of Medicine, China
}

ICA decreases myocardial hypertrophy injuries induced by isoproterenol by inhibiting endoplasmic reticulum stress Juan Luo,Yan Tan,Longrui Pan, Qiufang Zhang

(Department of Pharmacology,Hubei University of Medcine,Shiyan,China)

Abstract: Backgrounds and objective:Endoplasmic reticulum (ER) stress has been reported to be involved in many cardiovascular diseases such as hypertension, diabetes and atherosclerosis,myocardial ischemia,even the end stage of heart disease as heart failure.A number of studies show that Endoplasmic reticulum stress plays a critical role in the pathogenesis of heart failure.Pressure overload induced cardiac myocyte apoptosis is shown to be associated with increased endoplasmic reticulum stress in the mouse myocardium. Icariin (ICA), a natural flavonoid glucoside isolated from Epimedium brevicornum Maxim, has been proven to have various pharmacological activities. This study was undertaken to investigate whether ICA can protect from hypertrophy injuries induced by isoproterenol (ISO), and whether its mechanism is related to inhibiting endoplasmic reticulum stress.Methods:Myocardial hypertrophy injuries model was established by incubating $\mathrm{H} 9 \mathrm{c} 2$ cells in 100M M isoproterenol (ISO).H9c2 cells were randomly assigned to the control group,ISO group(100 M M for 24h), ICA+ISO group(treatment with 10,20,40 MM ICA and $100 \mathrm{MM}$ ISO for 24h, respectively),4-PBA group (100 \&MM 4-PBA +100 M M ISO for 24h).Hypertrophy was estimated by measuring the cell surface area,cell viability and ROS was determined by the MTT assay and fluorescent intensity in the endoplasmic reticulum. Apoptosis was measured using Hoechst 33258 staining and transmission electron microscopy.ERS signal pathway protein and apoptosis factors were analyzed using Western blot analysis.Results:ICA effectively suppress the H9c2 cells hypertrophy and apoptosis induced by ISO characterized by reducing surface area and LDH leakage and apoptotic rate and increasing protein amount.ICA decreased the production of reactive oxygen species (ROS) in endoplasmic reticulum,while promoting apoptosis protein Caspase-3,Bax and the endoplasmic reticulum signaling pathway protein GRP78,GRP94,IRE-1a,ATF6, PERK,cleaved XBP-1,P-eIF2a and CHOP was inhibited by icaiin. Conclusions:ICA treatment effectively suppressed H9C2 hypertrophy and apoptosis,its mechanism was related to inhibiting ROS production and endoplasmic reticulum stress.

*Key words:Icariin; Isoproterenol; Hypertrophy; Endoplasmic reticulum stress (ERS)

*This project was supported by the National Science Foundation of China (No:81641140,No:81303254)and Hubei Province Health and Family Planning scientific research project(No:WJ2017M214) 